\title{
PREFACE: HEAT AND MASS TRANSFER ENHANCEMENT ON MACRO-, MICRO-, AND NANOSCALES
}

This special issue is dedicated to the 25-year anniversary of the Journal of Enhanced Heat Transfer (JEH) and contains 3 papers in the field of heat and mass transfer intensification on the macro-, micro-, and nanoscales:

- "Choice and Justification of the Heat Transfer Enhancement Methods," by Yu. KuzmaKichta and A. Leontiev (this paper is based on Chapter 1 of the book, Heat and Mass Transfer Enhancement on Macro-, Micro-, and Nanoscales, by B. Dzyubenko, Yu. KuzmaKichta, A. Leontiev, I. Fedik, and L. Kholpanov, published by Begell House);

- "Vortex Heat Transfer Enhancement in Narrow Channels with a Single Oval-Trench Dimple Oriented at Different Angles to the Flow," by S. Isaev, A. Leontiev, Y. Chudnovsky, and I. Popov;

- "Heat and Mass Transfer Enhancement in Laminar Forced Convection Wet Channel Flows with Uniform Wall Heat Flux," by V.I. Terekhov, H.Q. Khafaji, and M.V. Gorbachev.

The methods of heat and mass transfer intensification have already been widely applied in the elements of power equipment, and they are very important. So the 25-year anniversary of JEH is a bright alignment and Begell House performs very important work for humanity!

This special issue is the survey of investigations in the field of heat and mass transfer enhancement on the macro-, micro-, and nanoscales. The available investigations into heat transfer and hydrodynamics of dimpled surfaces, helically profiled pipes, channels with knurling and microporous inserts are considered in detail. Consideration is given to the thermohydrodynamics on the micro- and nanoscales: heat transfer under condensation on macro- and microrough surfaces, heat transfer with boiling on surfaces with microporous coating and protrusions that form a homogeneous relief, heat transfer under convection in microchannels, the appearance of slipping on the wall in liquid flow over an ultrahydrophobic surface, and the influence of molecular layers of surfactant formed on surfaces on the hydraulic resistance of pipelines.

There are many passive techniques of heat transfer enhancement. However, only a few of them are capable of providing a reliable increase in a heat transfer rate exceeding an increase in pressure losses. One such successful technique is the profiling of a heat transfer surface with a regular arrangement of dimples. Consideration is given on detailed numerical simulation of fluid flow and heat transfer in the dimpled narrow channel at a Reynolds number of 10,000 . The orientation of oval-trench dimples on the heated surface to achieve superior thermal and hydraulic efficiency over spherical dimples is established. 
Evaporative cooling methods are among the most effective in air conditioning systems, so finding new ways to improve their performance is a relevant and important task. A new fundamental problem about the possibility of controlling the evaporation process with the help of heat supply (removal) from the wall in the direct evaporation cycle is considered. Such data are necessary for the proper organization of the cooling process. Consideration is given to intensification of heat and mass transfer in the cells of direct evaporative air cooling in the case of an additional supply of heat to the cell walls, but the surfaces of which evaporate the liquid. The problem is solved by a numerical method. Two-dimensional stationary Navier-Stokes, energy, and diffusion equations were solved for a laminar regime of flow. The effect of the Reynolds number $(\operatorname{Re}=50 / 1000)$, temperatures $\left(T_{0}=10 / 40^{\circ} \mathrm{C}\right)$, and humidity of air $\left(\varphi_{0}=0 / 50 \%\right)$ on an entrance and values of a thermal flux on a wall $\left(q^{*}=-0.1 /+0.2\right)$ for flow rate evaporation of a wet wall was studied. It is shown that vapor concentration and the temperature of air at a channel exit increase with an increase in an additional thermal flux at a wall. Thus, a normal vapor mass flux on surfaces also grows, which speaks to a more effective process of wall drying. In the paper, an analogy of processes of heat and mass transfer and friction on a surface with phase transitions and in the presence of an additional supply of heat to an evaporating surface is shown. The results of calculations are discussed in detail and practical recommendations on the optimization of evaporating cooling are given.

\title{
Guest Editors:
}

Professor Yury Kuzma-Kichta

Moscow Power Engineering Institute

Moscow, Russia

\author{
A. Leontiev \\ Bauman Moscow State Technical University \\ Moscow, Russia
}

